Research Paper

\title{
A Disposable Microfluidic Device for Controlled Drug Release from Thermal-Sensitive Liposomes by High Intensity Focused Ultrasound
}

\author{
Long Meng ${ }^{1 *}$, Zhiting Deng ${ }^{*}$, Lili $\mathrm{Niu}^{1 *}$, Fei $\mathrm{Li}^{1}$, Fei Yan ${ }^{1}$, Junru $\mathrm{Wu}^{2}$, Feiyan Cai ${ }^{\circledR}$ and Hairong Zheng ${ }^{1 凶}$ \\ 1. Paul C. Lauterbur Research Center for Biomedical Imaging, Institute of Biomedicaland Health Engineering, Shenzhen Institutes of Advanced \\ Technology, Chinese Academy of Sciences, Shenzhen, P.R. China, 518055 \\ 2. Department of Physics, University of Vermont, USA \\ *These authors contributed equally to this work.
}

$\triangle$ Corresponding authors: H. Z. (hr.zheng@siat.ac.cn) or F. C. (fy.cai@siat.ac.cn)

() 2015 Ivyspring International Publisher. Reproduction is permitted for personal, noncommercial use, provided that the article is in whole, unmodified, and properly cited. See http://ivyspring.com/terms for terms and conditions.

Received: 2015.04.01; Accepted: 2015.07.15; Published: 2015.08.08

\begin{abstract}
The drug release triggered thermally by high intensity focused ultrasound (HIFU) has been considered a promising drug delivery strategy due to its localized energy and non-invasive characters. However, the mechanism underlying the HIFU-mediated drug delivery remains unclear due to its complexity at the cellular level. In this paper, micro-HIFU (MHIFU) generated by a microfluidic device is introduced which is able to control the drug release from temperature-sensitive liposomes (TSL) and evaluate the thermal and mechanical effects of ultrasound on the cellular drug uptake and apoptosis. By simply adjusting the input electrical signal to the device, the temperature of sample can be maintained at $37^{\circ} \mathrm{C}, 42^{\circ} \mathrm{C}$ and $50{ }^{\circ} \mathrm{C}$ with the deviation of $\pm 0.3{ }^{\circ} \mathrm{C}$ as desired. The flow cytometry results show that the drug delivery under MHIFU sonication leads to a significant increase in apoptosis compared to the drug release by incubation alone at elevated temperature of $42{ }^{\circ} \mathrm{C}$. Furthermore, increased squamous and protruding structures on the surface membrane of cells were detected by atomic force microscopy (AFM) after MHIFU irradiation of TSL. We demonstrate that compared to the routine HIFU treatment, MHIFU enables monitoring of in situ interactions between the ultrasound and cell in real time. Furthermore, it can quantitatively analyze and characterize the alterations of the cell membrane as a function of the treatment time.
\end{abstract}

Key words: ultrasound-mediated drug delivery; high intensity focused ultrasound; mechanical and thermal effects; microfluidics

\section{Introduction}

Although various new therapeutic innovations such as gene therapy and Immunotherapy are being developed, chemotherapy remains the primary choice of treatment for a variety of cancers [1]. The success of chemotherapy is not only related to the efficacy of the drug but also to the concentration of the drug attained in tumors. The aim of targeted drug delivery is to increase the concentration of a chemotherapeutic agent at the site of disease while minimizing the side effects to the neighboring normal cells. In other words, safe and efficient delivery of therapeutic dose of drug to the interior of the cell membrane is critical to the success of cancer chemotherapy.

Nano-scale liposomes, due to their small size and long circulation time in tumor blood vessels, have been used to preferentially deliver chemotherapeutic 
agents into tumors and maintain a high concentration in situ [2-4]. Furthermore, drugs entrapped in liposomes can be released in response to diverse stimuli such as variation in $\mathrm{pH}$, electromagnetic radiation (light) excitation, local enzymes and heat enhancement [5-8]. Heat-responsive drug delivery, which takes advantage of low-melting point of membrane lipids, has attracted increasing attention because of its low-toxicity [9, 10]. When the local temperature reaches the phase-transition temperature of lipid bilayers between $41{ }^{\circ} \mathrm{C}$ and $43{ }^{\circ} \mathrm{C}$, the membrane of temperature-sensitive liposomes (TSL) undergoes a phase transition from a gel phase to a liquid crystalline phase. The permeability of the membrane increases significantly to promote the release of the encapsulated drugs from liposomes. However, the external excitation with electromagnetic radiation requires the insertion of an interstitial needle or an antenna into tissues, limiting its applications because of the invasive nature.

Ultrasound is a mechanical wave of frequency above $20 \mathrm{kHz}$. The high intensity focused ultrasound (HIFU) can influence the cellular activity by its localized thermal and mechanical effects [11]. For its thermal effect, HIFU generated by an extracorporeal ultrasound transducer can induce local temperature

A
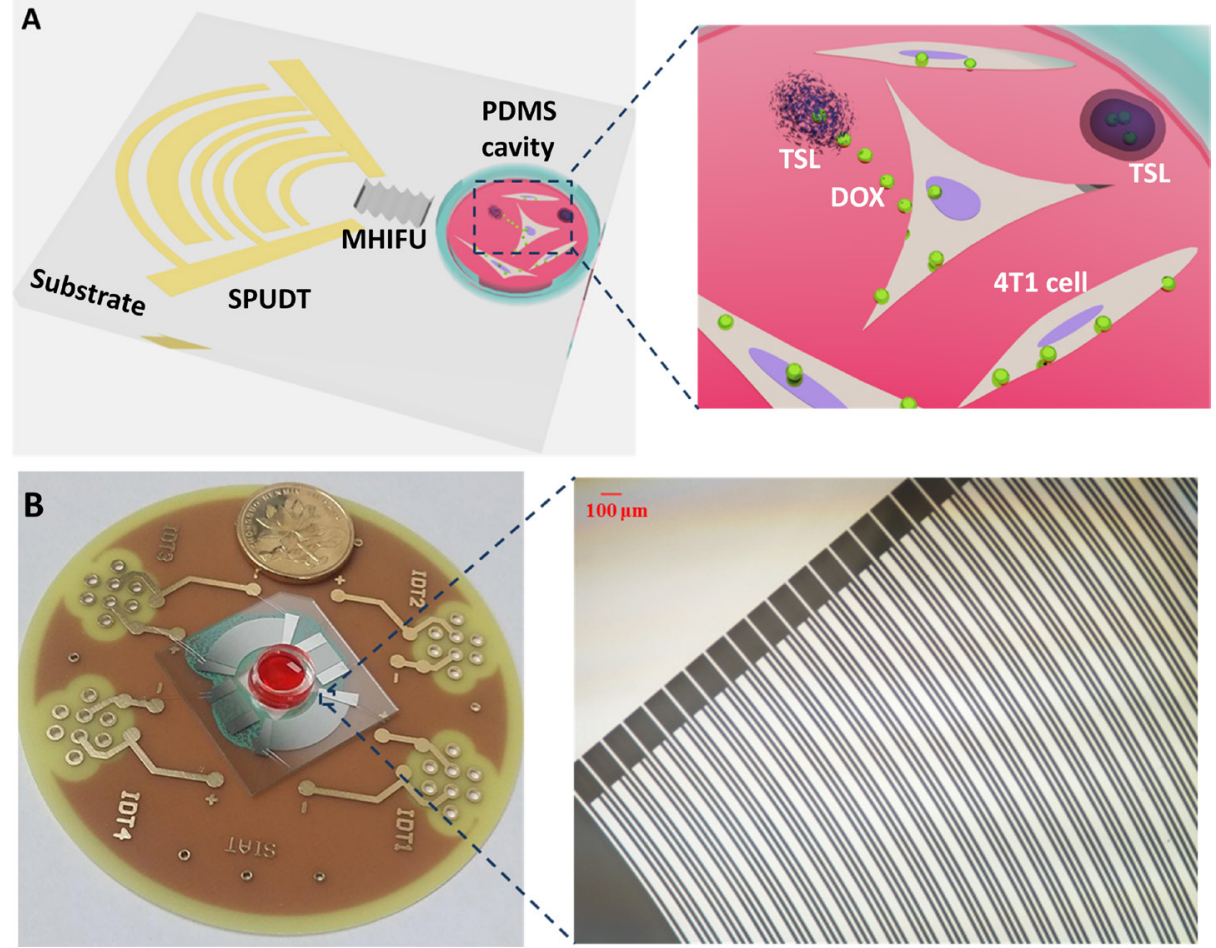

Figure 1. (A) Schematic of the MHIFU device and MHIFU-mediated TSL release. Device architecture shows the SPUDT with curved electrodes to generate the focused SAW on the LiNbO3 piezoelectric wafer. The MHIFU is coupled to the PDMS cavity. Inset: the encapsulated drug is released from TSL due to the mechanical and thermal effects of MHIFU. The drug is then internalized by 4T1 cells cultured in PDMS cavity. (B) Optical imaging of MHIFU device. Inset: The finger pairs of the SPUDT on the substrate. The MHIFU was fabricated by deposition of circular electrodes on a $1 \mathrm{~mm}$ thickness $128^{\circ} \mathrm{Y}$-rotated, $\mathrm{X}$-propagating LiNbO3 substrate. In all experiments, only one SPUDT was excited to generate the MHIFU. rise inside the human body and trigger the drug release from TSL. It therefore has the potential to be a noninvasive modality for clinical applications [12-17]. However, the complex and microscopic interactions between ultrasound and cells have proven to be difficult for revealing the mechanism of HIFU-mediated drug delivery.

Recently the surface acoustic wave (SAW)-based microfluidic device, has been widely used in various applications, such as particle [18-23] and fluid [24-26] manipulation, atomization[27, 28], as well as sonoporation[29] because of its following capabilities: localization of acoustic energy along the substrate surface; high operating frequency $(\geq 20 \mathrm{MHz})$; standard micro-fabrication process and excellent optical transparency of the device.

To distinguish the conventional HIFU, we name the high intensity focused ultrasound generated by a SAW-based microfluidic device as micro-HIFU (MHIFU). In this paper, we use MHIFU to investigate its thermal and mechanical effects on the mechanism of TSL release (Fig. 1A). Using this microfluidic device, we can accurately control the localized temperature elevation within a deviation of approximately \pm $0.3^{\circ} \mathrm{C}$. Furthermore, the cellular uptake and apoptosis induced by MHIFU-mediated drug delivery at various temperatures are quantitatively measured by flow cytometry. The structure of cell membranes is imaged by an atomic force microscope (AFM), providing a microscopic insight on the mechanism of MHIFU-mediated drug delivery.

\section{Materials and Methods}

\section{Fabrication of MHIFU de- vice and PDMS cavity}

The source of MHIFU used in this study is a single element unidirectional transducer (SPUDT) with curved electrodes to concentrate the acoustic energy into a focused zone. Besides enhancing acoustic energy in the focused zone, SPUDT reduces the loss of acoustic energy due to bidirectional propagation. The resonant frequency of the circular SPUDT was designed to $30 \mathrm{MHz}$ with an aperture angle of $90^{\circ}$ The MHIFU was 
fabricated by deposition of circular electrodes with 40 pairs on a $1 \mathrm{~mm}$ thickness $128^{\circ}$ Y-rotated, X-propagating $\mathrm{LiNbO}_{3}$ substrate (Fig. 1B). In all experiments, only one SPUDT was excited to generate the MHIFU.

Fabrication of PDMS cavity mainly included three steps. First, Polydimethylsiloxan (PDMS, Sylgard 184, Dow Corning, USA) mixed at a 10:1 curing ratio was spin coated onto a silicon wafer and then was cured at $80^{\circ} \mathrm{C}$ for 30 min to obtain a thin PDMS film. The thickness of the PDMS film, characterized by a step profiler (XP-1, MTS, USA), was about $120 \mu \mathrm{m}$. Then, a circular opening (diameter, $8 \mathrm{~mm}$ ) was drilled through a blank bulk PDMS with a height of $3 \mathrm{~mm}$. Finally, the bulk PDMS with openings was bonded to the PDMS film by the plasma treatment; the PDMS film could then be peeled off from the silicon wafer to form multiple PDMS cavities. The single PDMS cavity could be isolated using a $10 \mathrm{~mm}$ puncher (Harris Uni-Core, World Precision Instruments, USA) and had a diameter of $8 \mathrm{~mm}$ with a height of $3 \mathrm{~mm}$. The PDMS cavity was placed on the $\mathrm{LiNbO}_{3}$ substrate directly and the centre of the PDMS cavity was located at the focal position of MHIFU.

\section{Cell culture and temperature sensitive lipo- some (TSL) preparation}

Mouse 4T1 breast tumor cells, a typical model of cancer cells, were used in the experiment to investigate the response of cells to thermal effects of MHIFU. Prior to seeding the cells in PDMS cavity, the surface of PDMS film was coated with poly-L-lysine to enhance the dispersion and adhesion of cells. The 4T1 cells in the PDMS cavity were cultured in Dulbecco's Modified Eagle's Medium (DMEM, Gibco, USA) supplemented with $10 \% \mathrm{v} / \mathrm{v}$ fetal bovine serum (FBS; Life Technologies Corporation, USA) at $37^{\circ} \mathrm{C}$ with $5 \%$ carbon dioxide $\left(\mathrm{CO}_{2}\right)$.

The TSL formulation was composed of DPPC: MPPC: DSPE-PEG-2000 in the molar ratio of 86:10:4. Encapsulation of Doxorubicin (DOX) into the liposomes was carried out using a remote loading protocol [30]. Free DOX was removed by ultrafiltration (MWCO, 100 000). DOX encapsulation efficiency (EE) was calculated by comparing the total fluorescence intensity of DOX before and after purification. \% $E E=I(t)$ after purification/I(t) before purification* 100 , where $I(t)$ is the fluorescence intensity of liposome suspension after liposome lysis with 1\% Triton X-100. The DOX concentration was determined by fluorescence intensity measurements $(\lambda \mathrm{ex}=485 \mathrm{~nm}, \lambda \mathrm{em}=$ $550 \mathrm{~nm}$ ). The size and polydispersity of liposome was $79.51 \mathrm{~nm}$ (Additional File 4: Fig. S1) determined by dynamic light scattering (Zetasizer Nano ZS, Malvern
Instrument, UK). Characterization of TSL is listed in Additional File 4: Table S1.

\section{Experimental system}

To characterize the performance of the MHIFU, an in situ real-time monitor was setup to measure the thermal effects of ultrasound using the optical imaging and infrared thermal imaging methods. The MHIFU was mounted on a stage of an inverted fluorescence microscope (DMI3000B, Leica, Germany) to investigate the intracellular uptake and distribution of DOX in cells. The thermal distribution of fluid medium in the PDMS cavity was visualized by a thermal infrared imager (T440, FLIR, USA). Sinusoidal signals of $30 \mathrm{MHz}$ were produced by a signal generator (AFG 3102, Tektronix, USA), amplified by a power amplifier (ZHL-1-2W, MiniCircuits, German), and then applied to the SPUDT. The continuous wave (CWs) was initially applied to SPUDT for temperature elevation. Subsequently, the SPUDT was switched to a burst mode (duty cycle $=28.6 \%, 52.8 \%, 79.2 \%$ ) to maintain the fluid temperature in PDMS cavity at $37^{\circ} \mathrm{C}, 42{ }^{\circ} \mathrm{C}$, and $50{ }^{\circ} \mathrm{C}$. In all experiments, the peak to peak electronic voltage from the output of the power amplifier applied to MHIFU was kept constant at $20 \mathrm{~V}_{\mathrm{p}-\mathrm{p}}$. The acoustic pressure at the MHIFU focus was about 0.68 MPa measured by a calibrated needle hydrophone (Precision Acoustics, Dorchester, Dorset, UK) which was positioned $100 \mu \mathrm{m}$ above the substrate.

\section{Atomic force microscopy (AFM) imaging}

The cells after MHIFU irradiation with TSL in the PDMS cavity were treated by glutaraldehyde $(2.5 \%, 10 \mathrm{~min})$, washed by PBS and then air-dried. AFM imaging was performed on a MFP-3D bio-atomic force microscope (Asylum Research, Santa Barbara, CA) operated in a contact mode. The topographic images of the membrane were acquired at a scan field of $5 \mu \mathrm{m} \times 5 \mu \mathrm{m}$.

\section{Measurement of drug release from TSL lipo- somes}

DOX liposomes were diluted to a final concentration of $0.1 \mu \mathrm{g} / \mathrm{mL} .100 \mu \mathrm{l}$ of the prepared liposomal solution was added to the PDMS cavity. When the temperature of the solution reached $37^{\circ} \mathrm{C}, 42^{\circ} \mathrm{C}$ and $50^{\circ} \mathrm{C}$ by the aforementioned CWs, it was maintained at the desired temperatures for $0.33,0.67,1,3,5,8$, and $10 \mathrm{~min}$. Subsequently, samples of the liposomal solution were transferred from PDMS cavity into black 96-well plates and the fluorescence intensity of DOX in each well was measured with a microplate reader (Synergy 4, BioTek, USA) at excitation and emission wavelengths of 485/550 nm. Meanwhile, an amount of $100 \mu \mathrm{l}$ liposomal solution was also heated in an incubator and maintained for $0.33,0.67,1,3,5,10,20$, 
and $30 \mathrm{~min}$ at $37^{\circ} \mathrm{C}, 42{ }^{\circ} \mathrm{C}$ and $50^{\circ} \mathrm{C}$. The percentage of DOX release at different temperatures and durations was calculated using the following equation:

$$
\% \text { release }=\frac{I_{t}-I_{0}}{I_{100}-I_{0}} \times 100 \%
$$

where $I_{t}$ is the fluorescence intensity of a sample at time point $t ; I_{0}$ is the steady fluorescence intensity of a sample measured at room temperature $\left(26{ }^{\circ} \mathrm{C}\right) ; I_{100}$ was the intensity after the addition of detergent Triton X-100.

\section{Evaluation of drug uptake and cell apoptosis}

After irradiation by MHIFU as described above, the cells in the PDMS cavity were transferred back to the incubator at $37^{\circ} \mathrm{C}$ for $30 \mathrm{~min}$. The qualitative and quantitative characterization of DOX uptake of cells was carried out by laser scanning confocal microscopy (TCS SP5, Leica, Germany) and flow cytometery (Accuri C6, BD Biosciences, USA) respectively. For the confocal microscopy, the cells were fixed in $4 \%$ formaldehyde for $10 \mathrm{~min}$, permeabilized with $1 \%$ Triton X-100 for 5 min and then blocked with 2\% BSA for 30 min. The cells were then incubated with Alexa Fluor ${ }^{\circledR}$ 488 Phalloidin (Molecular probes, USA) for $20 \mathrm{~min}$ at room temperature to stain F-actin. After washing with PBS, the cells were counterstained with DAPI for 15 min. For flow cytometery study, at least 10,000 events were counted to measure the mean fluorescent intensity determined from a flow cytometry histogram.

Prior to investigating the cell apoptosis, TSL was added to 4T1 cells for MHIFU irradiation or incubation treatment for $3 \mathrm{~min}$. Then, the TSL and 4T1 cells were co-cultured for $30 \mathrm{~min}$. Subsequently, the TSL was removed, washed with PBS and the cells were further cultured for $4 \mathrm{~h}$. The apoptosis detection was carried out after $4.5 \mathrm{~h}$ using cells irradiated by MHIFU and TSL. The cells were harvested and labeled fluorescently to detect the apoptosis at early stage or late stage by adding the binding assay of Annexin V- fluorescein isothiocyanate (FITC)/ Prodium Iodide (PI) kit (BD PharMingen, San Diego, USA) to each sample. Annexin V and PI emissions were detected in the FL-1 (band pass $530 \mathrm{~nm}$, bandwidth $30 \mathrm{~nm}$ ) and FL-2 (band pass $585 \mathrm{~nm}$, bandwidth $42 \mathrm{~nm}$ ) channels, respectively.

\section{Measurement of flow field of acoustic stream- ing and shear stress}

To obtain velocity data and visualize the flow field within the PDMS cavity actuated by MHIFU, a particle image velocimetry (PIV) study was carried out. PIV provides an effective means of quantitatively characterizing the velocity distribution of the complex field [31]. The polystyrene particles (Sigma-Aldrich,
Missouri USA) with the diameter of $10 \mu \mathrm{m}$ were chosen as the tracer particles, and a video of the acoustic streaming was captured by a high speed charge coupled device (CCD) camera (MC1310, Mikrotron, Germany) at 500 frames/s. The shear stress induced by the gradient of acoustic streaming velocity could be estimated [32]:

$$
\tau=\eta \sqrt{\left(\frac{\partial u}{\partial y}\right)^{2}+\left(\frac{\partial v}{\partial x}\right)^{2}}
$$

where $\eta$ is the viscosity of fluid; $u$ and $v$ are the flow velocity in $x$ and $y$ directions, respectively.

\section{Results and discussion}

\section{Mechanical and thermal effects of MHIFU}

MHIFU is designed to generate a strong acoustic radiation force to drive a droplet movement on the surface of the substrate. Two $\mu$ l of pure water was placed at the focus of MHIFU directly. When we applied CWs sinusoidal electric voltage with the amplitude of $20 \mathrm{~V}_{\mathrm{p} \text {-p }}$ to SPUDT, the $2 \mu \mathrm{l}$ of pure water moved along the MHIFU propagation direction immediately and the average translational velocity was $20 \mathrm{~mm} / \mathrm{s}$ (also see Additional File 1: Movie S1).

To trigger the TSL release, it is crucial to accurately regulate the temperature of the sample. A semi-open PDMS cavity with the diameter of $8 \mathrm{~mm}$ was placed at the focal region of MHIFU and $100 \mu \mathrm{l}$ deionized water was injected into the cavity. Accurate controlling of the temperature at desired degrees involved two stages: (1) temperature elevation period and (2) temperature maintenance period. A continuous radio frequency (RF) signal was applied to a single SPUDT to heat the fluid continually while RF pulse signal was utilized to maintain the temperature at desired degrees by adjusting the pulse length and pulse repetition frequency (PRF). Fig. 2A shows the evolution and spatial distribution of fluid temperature in PDMS cavity as a function of time (also see Additional File 2: Movie S2). The distribution of the sample in PDMS cavity is visualized by a thermal infrared imager in real time. The results show that the temperature of sample in PDMS cavity increased quickly from $26^{\circ} \mathrm{C}$ to $42{ }^{\circ} \mathrm{C}$ within less than one minute when the CWs with the voltage of $20 \mathrm{Vp}$-p was applied to the SPUDT. To maintain the sample temperature at $42{ }^{\circ} \mathrm{C}$, the SPUDT was operated with burst RF signal (frequency $=30 \mathrm{MHz}$, length of each burst = $1.6 \mathrm{k}$ cycles, $\mathrm{PRF}=10 \mathrm{kHz}$, and the peak-to-peak electric voltage $=20 \mathrm{~V}$ ). As shown in Fig. $2 \mathrm{~A}$, the temperature of sample was kept constant at $42{ }^{\circ} \mathrm{C}$ accurately for $5 \mathrm{~min}$ and the temperature deviation was approximately within $\pm 0.3{ }^{\circ} \mathrm{C}$. In both heating and maintaining temperature processes, the temperature 
distribution of fluid in PDMS cavity was relatively uniform. The sample temperature could reach $60{ }^{\circ} \mathrm{C}$ or even higher by increasing the input power and a longer duration. In addition, Fig. 2B shows the temperature variation in PDMS cavity as a function of time at the desired temperature of $37^{\circ} \mathrm{C}, 42^{\circ} \mathrm{C}$ and $50^{\circ} \mathrm{C}$. This demonstrates that the temperature of fluid in PDMS cavity remained nearly constant (at various desired temperatures) through a time duration.

\section{MHIFU-mediated release of drug from the TSL}
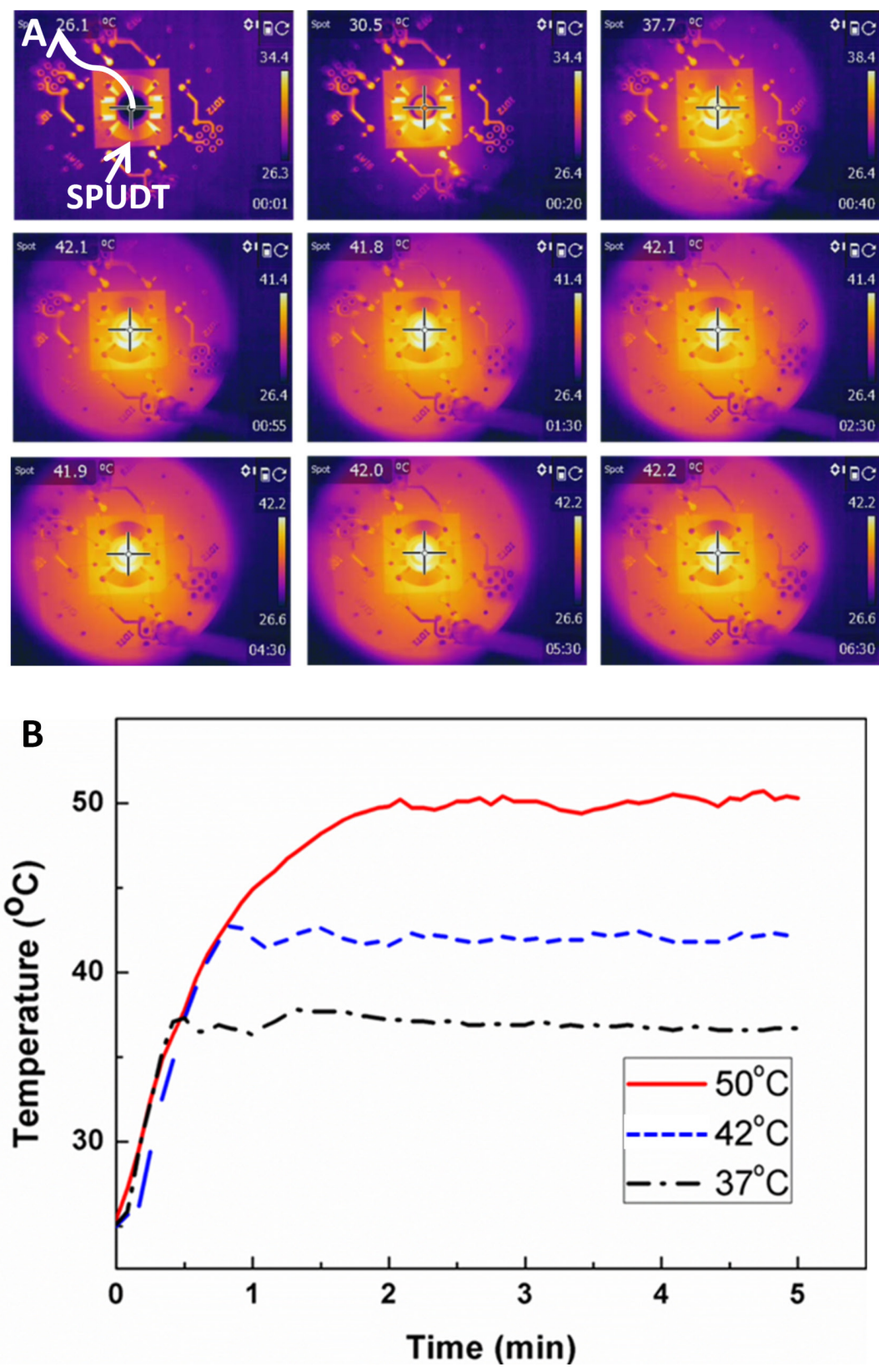

Figure 2. (A) Evolution and the spatial distribution of the fluid temperature in PDMS cavity at as a function of time. Additional File 2: Movie S2 shows the temperature variation with the time induced by MHIFU ( $\left.30 \mathrm{MHz}, 20 \mathrm{~V}_{\mathrm{p}-\mathrm{p}}\right)$ at five times the normal speed. (B) The temperature of fluid in PDMS cavity can be elevated and maintained at $37^{\circ} \mathrm{C}, 42^{\circ} \mathrm{C}$ and $50^{\circ} \mathrm{C}$ precisely by adjusting the input pulse length and PRF.
Prior to investigating the efficiency of ultrasound mediated drug release from TSL, it is essential to be aware of the temperature sensitivity of TSL. The TSL samples were heated in a thermostatically controlled dry bath (Labnet International, Rutland, UK) to make the sample temperature more uniform and stable. The dashed lines in Fig. 3A show the efficiency of drug release of sample in an incubator at various temperatures as a function of time. After 10 min of incubation, the release rate was $16.7 \% \pm 7.3 \%$ and $79.8 \% \pm 4.5 \%$ at $37^{\circ} \mathrm{C}$ and $50^{\circ} \mathrm{C}$, respectively. When the temperature remained at $42{ }^{\circ} \mathrm{C}$, the release rate increased from $47.6 \% \pm 4.2 \%$ to $64.4 \% \pm$ $6.2 \%$ as the time of incubation increased from $10 \mathrm{~min}$ to $20 \mathrm{~min}$. The results indicate that the efficiency of DOX release increased with the increment of temperature and duration of incubation. Increase in temperature initiates the phase transition of TSL, rendering a higher permeability of the liposome membrane for releasing the DOX, indicating that the TSL is sensitive to the temperature elevation.

The efficiency of MHIFUmediated drug release is shown by the solid lines in Fig. 3B. The results show that in analogy to the time of incubation, the rate of drug release from TSL increases as the temperature is elevated. However, the drug release from TSL irradiated by MHIFU was much faster than that of the incubation at 42 ${ }^{\circ} \mathrm{C}$. After $3 \mathrm{~min}$ of ultrasonic irradiation, the efficiency of drug release attained $55.2 \% \pm 5.9 \%$ at $42{ }^{\circ} \mathrm{C}$, which was much higher than that of incubation at $42{ }^{\circ} \mathrm{C}$ of $31.3 \% \pm 7.1 \%$. The increased efficiency of drug release mainly contributed to the local temperature elevation and mechanical effects of MHIFU. When the SAW approached the boundary of the PDMS, part of the energy of the SAW was absorbed by PDMS boundary. The rest of acoustic energy existed in the leaky SAW (LSAW) and longitudinal bulk wave diffracted under an angle $\theta R$ into the fluid[33]. Since the thickness of the PDMS boundary was about $2 \mathrm{~mm}$, the actuation of acoustic energy in PDMS boundary was relatively small and the most acoustic energy was coupled with the confined fluid in the PDMS cavity. Due to the attenuation of longitude 
bulk wave, most acoustic energy was converted to the acoustic streaming leading to internal streaming in the PDMS cavity. The acoustic streaming in PDMS cavity induced by MHIFU may play an important role in triggering drug release from TSL. Fig. 3C shows the streamline of flow field in the PDMS cavity when the SPUDT was operated in a burst mode $(2.6 \mathrm{k}$ cycles, 3.3 $\mathrm{kHz}$ PRF) to maintain the temperature at $37^{\circ} \mathrm{C}$. The pattern of the streamline illustrated that the acoustic streaming was vortex-type and the maximum rotational speed was measured to be $1.6 \mathrm{~mm} / \mathrm{s}$ using PIV method (also see Additional File 3: Movie S3). Fig. 3D shows the distribution of shear stress induced by acoustic streaming and the maximum stress is about 0.26 dyne/cm2. In addition, no significant improvement of drug release was obtained by increasing the duration of irradiation for more than $3 \mathrm{~min}$ at $42{ }^{\circ} \mathrm{C}$. Thus, the duration of MHIFU irradiation was determined to be $3 \mathrm{~min}$.

\section{Uptake of drug released from TSL by 4T1 cells}

Figure 4A shows the uptake of DOX by $4 \mathrm{~T} 1$ cells at 30 min after MHIFU treatment. The red fluorescent of DOX can serve to assess the cellular uptake and distribution. The cellular myofilament and nuclei were stained with FITC-phalloidin and DAPI, giving green and blue fluorescence, respectively. When the fluid in PDMS cavity was heated by MHIFU at $37^{\circ} \mathrm{C}$, extremely weak DOX fluorescence signal can be found in the cellular nuclei. However, at $42{ }^{\circ} \mathrm{C}$, the cells emitted strong red fluorescence, which was colocalized with the blue DAPI signal indicating accumulation of DOX in the nuclei. As the temperature increased to $50{ }^{\circ} \mathrm{C}$, treated cells showed nuclear disintegration and myofilament fragmentation. Further experiments were carried out to quantitatively analyze cellular uptake of DOX by mean fluorescence intensities of cytometry histograms. As shown in Fig. $4 \mathrm{~B}$, the rate of cellular uptake increased with the increment of temperature. For $37^{\circ} \mathrm{C}$ and $50{ }^{\circ} \mathrm{C}$, there was no significant difference between incubation and MHIFU irradiation. However, the mean fluorescence intensity generated by MHIFU irradiation was 1.5 fold higher than that of incubation-induced drug release at $42^{\circ} \mathrm{C}$, suggesting MHIFU promoted the DOX uptake.
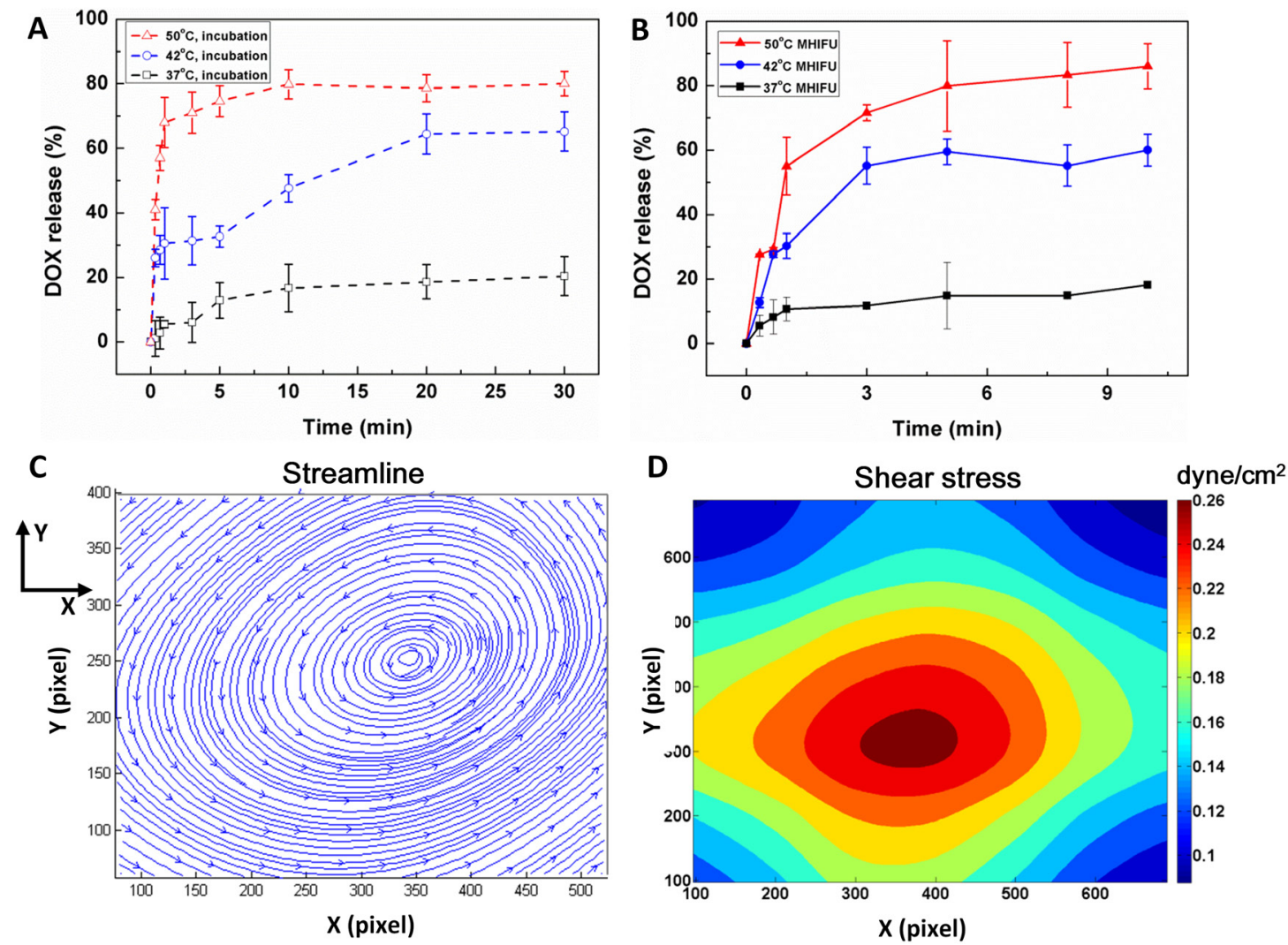

Figure 3. Temperature dependence of drug release from TSL induced by (A) MHIFU and (B) incubation. (C) The streamline of the flow field by PIV analysis. The streamline indicated the acoustic streaming in PDMS cavity was a kind of rotational streaming. The maximum and average rotational speed was $1.6 \mathrm{~mm} / \mathrm{s}$ and 743.8 $\mu \mathrm{m} / \mathrm{s}$ when the SPUDT was operated at a burst mode to maintain the temperature at $37^{\circ} \mathrm{C}$. (D) The distribution of shear stress induced by acoustic streaming. The maximum shear stress was about 0.26 dyne/ $\mathrm{cm}^{2}$. The MHIFU propagated along the negative $X$ axis. 


\section{Apoptosis induced by MHIFU-mediated drug release}

To investigate the cellular apoptosis induced by MHIFU-mediated drug release, the 4T1 cells were labeled with Annexin V-FITC/PI and analyzed by flow cytometry. The experiments were divided into

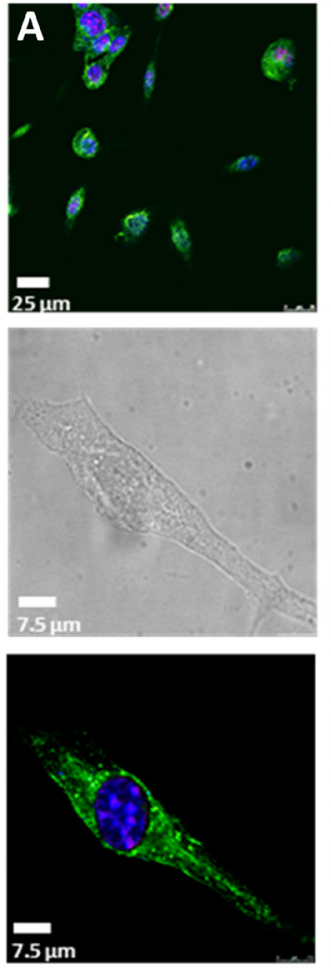

$37^{\circ} \mathrm{C}$

B

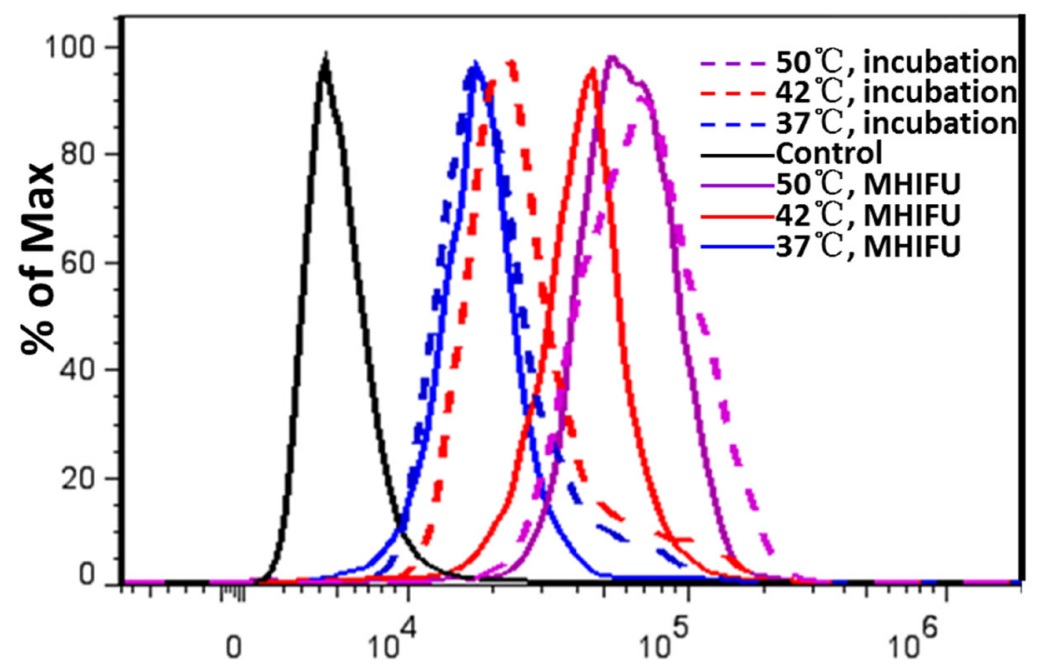

FL2-A

Figure 4. Qualitative and quantitative analysis of cellular uptake at various temperatures by confocal microscope and flow cytometry. (A) The fluorescence image shows the drug has entered into the cellular nuclei after MHIFU irradiation at $42{ }^{\circ} \mathrm{C}$ while the nuclear fragmentation and myofilament destruction were occurred at $50^{\circ} \mathrm{C}$. (B) Flow cytometry result shows the cellular uptake increases with the temperature elevation and cellular uptake irradiated by MHIFU at $42^{\circ} \mathrm{C}$ was 1.5 times larger than that of incubation. four groups: (1) MHIFU irradiation for $3 \mathrm{~min}$, in the presence of TSL; (2) MHIFU irradiation for $3 \mathrm{~min}$ solely; (3) incubation for 3 min together with TSL; (4) control group containing cells maintained at $37^{\circ} \mathrm{C}$ without treatment. For the first and third groups, after 30 min of incubation, the PDMS cavity was washed 3 times to remove the TSL not internalized by $4 \mathrm{~T} 1$ cells. The apoptosis detection was carried out at $4.5 \mathrm{~h}$ after the cells were treated by MHIFU or incubation. When the fluid temperature in PDMS cavity was held constant at $37{ }^{\circ} \mathrm{C}$, no significant apoptosis was found in any group compared to the control group. As shown in Fig. 5, about $92.7 \%$ and $93.1 \%$ cells maintained viability in the first and third group, respectively. However, as the temperature was increased to $42{ }^{\circ} \mathrm{C}$, late apoptotic cells in the first group reached up to $17.8 \%$, which was much higher than that of the second $(3 \%)$ and the third group $(3.5 \%)$. It is of note the cell viability in the second group was $94.3 \%$ suggesting that the short duration of MHIFU treatment alone could not exert an adverse effect on the cell viability. Lack of apoptosis in the third group at $42^{\circ} \mathrm{C}$ was probably due to the short heating duration (3 min). Moreover, dead cells in the first group increased up to $5.3 \%$ indicating that application of MHIFU in TSL delivery was capable of accelerating the apoptosis within a short duration of treatment. When the temperature in PDMS cavity reached $50{ }^{\circ} \mathrm{C}$, apoptotic cells in each group increased dramatically. It can be seen that high levels of apoptosis were achieved regardless of whether TSL were participated or not. This shows that the high temperature (50 ${ }^{\circ} \mathrm{C}$ ) is the dominant factor causing the apoptosis. In the MHIFU-mediated drug delivery, both cellular uptake and apoptosis are closely related to the temperature and increased with the temperature elevation, as shown in Fig. 6.

\section{Mechanism of MHIFU-mediated drug delivery}

HIFU-triggered release of drugs as a promising approach to realize the local drug delivery has attracted a great deal of attention from researchers and clinicians. Understanding the mechanism of ultrasound-mediated drug delivery not only 
provides an effective drug delivery method but also could be utilized for therapeutic and clinical applications. Cell morphology and shape after MHIFU irradiation with TSL could provide the information about cell activity and viability. Long-time monitoring of the changes in cell morphology showed that the cells experience shrinking, degenerating, detaching from the substrate and eventually released into the medium (Additional File 4: Fig. S2).

Ultrasound mediated drug delivery is a complex process and the study of its bioeffects at a sub-micron to nanometer scale may provide an important new insight into the underlying mechanisms. To investigate the cellular morphology, we used AFM to scan the surface of the cell membrane. Figure 7 shows the deflection and topography images of a fixed cell after the treatment of MHIFU combined with TSL at various temperatures. As shown in Fig. 7, the cell surface at $37^{\circ} \mathrm{C}$ and $42{ }^{\circ} \mathrm{C}$ is relatively smooth, and similar to the control group. The myofilament was intact and no pore-like structures were observed on the membrane. However, the cell surface shows more protruding structures at $50{ }^{\circ} \mathrm{C}$. The disorganized membrane surface and destroyed myofilament reveal that high temperature-induced cell damage occurred at $50{ }^{\circ} \mathrm{C}$. At $4 \mathrm{~h}$ after MHIFU-mediated drug delivery at $42{ }^{\circ} \mathrm{C}$, the cell surface showed somewhat squamous structure with more uniform size and some depressions. Protruding and depression structures with larger magnitude were more obvious as the time extended from $6 \mathrm{~h}$ to $10 \mathrm{~h}$. The changes in the morphology of the cellular membrane are probably due to the synergistic effect of MHIFU and drug. The flow cytometry results also show that the apoptotic cells in MHIFU-mediated drug delivery group is much larger than that of incubation-induced drug delivery group at $42{ }^{\circ} \mathrm{C}$. Therefore, besides thermal effects, the mechanical effects, such as acoustic streaming, play an important role in the process of the ultrasound-mediated drug
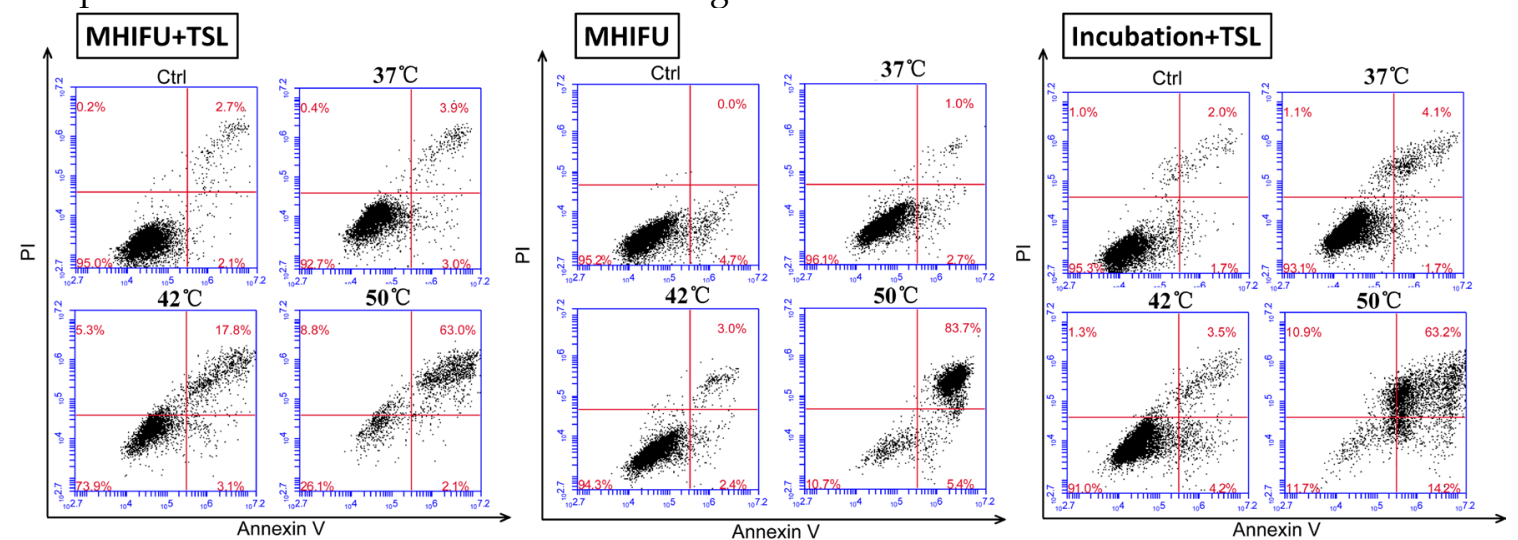

Figure 5. Quantitative analysis of apoptosis by flow cytometry. The cells were divided four groups: MHIFU-mediated drug delivery; MHIFU irradiation solely; water incubation-mediated drug delivery and control group. It can be seen that the cell did not show significant change in viability at $37{ }^{\circ} \mathrm{C}$ for each group. For $42{ }^{\circ} \mathrm{C}$, the late stage apoptotic cells were detected only in the first group, indicating MHIFU irradiation solely would not exert an adverse effect on the viability while the third group did not show obvious apoptosis. When the temperature increased to $50^{\circ} \mathrm{C}$, apoptosis in each group increased dramatically because of high-hyperthermia. 


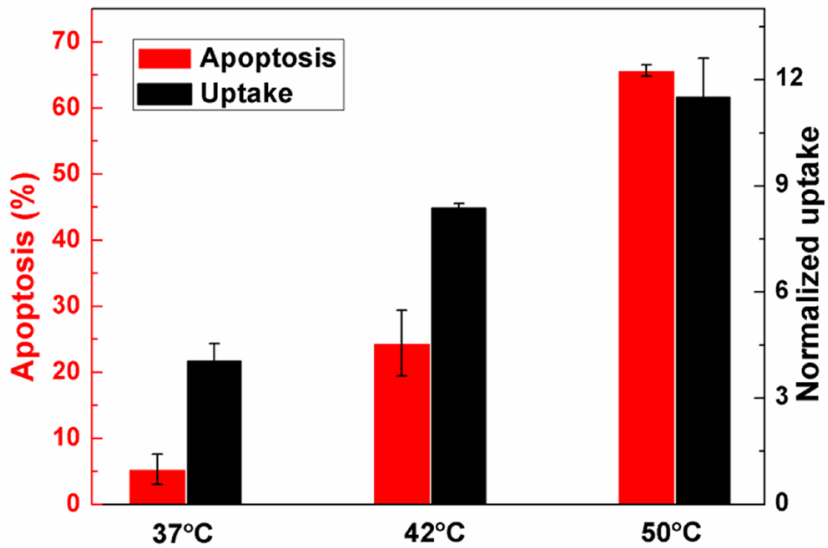

Figure 6. The relationship between SAW and cellular uptake and apoptosis at various temperatures. The cellular uptake is a normalized result.

\section{Control}
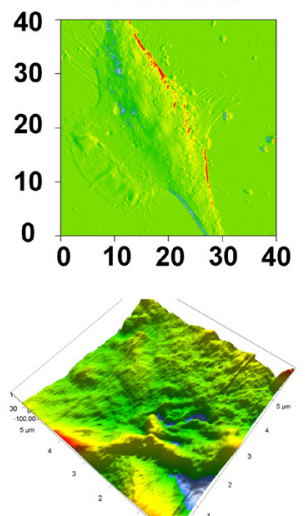

$42^{\circ} \mathrm{C}, 4 \mathrm{~h}$
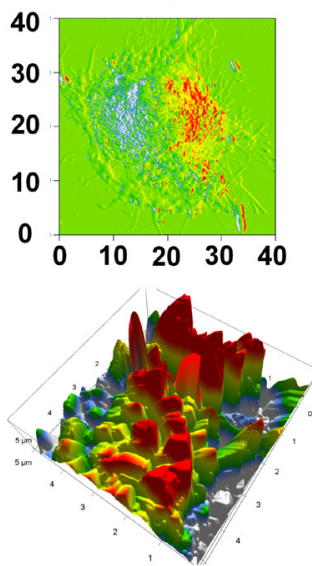

$37^{\circ} \mathrm{C}$
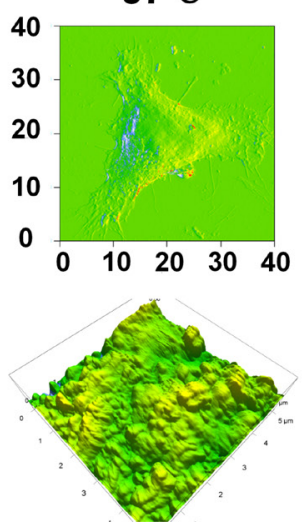

$42^{\circ} \mathrm{C}, 6 \mathrm{~h}$
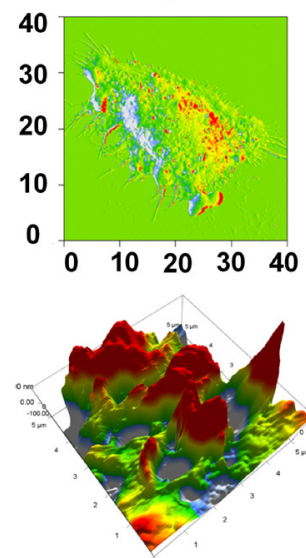

$42^{\circ} \mathrm{C}$
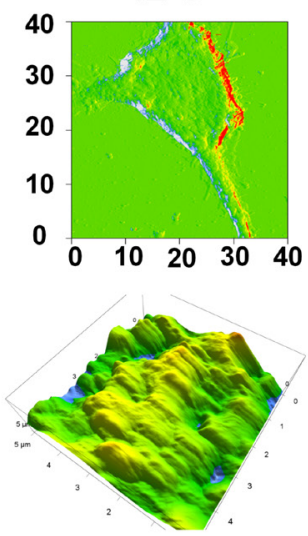

$42^{\circ} \mathrm{C}, 8 \mathrm{~h}$
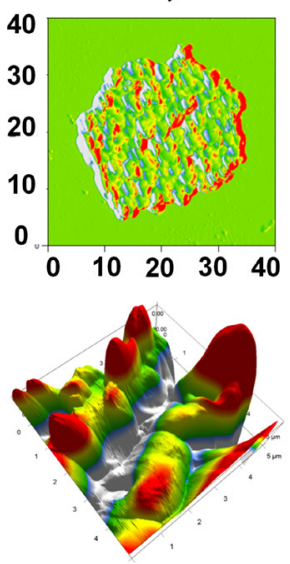

$50^{\circ} \mathrm{C}$
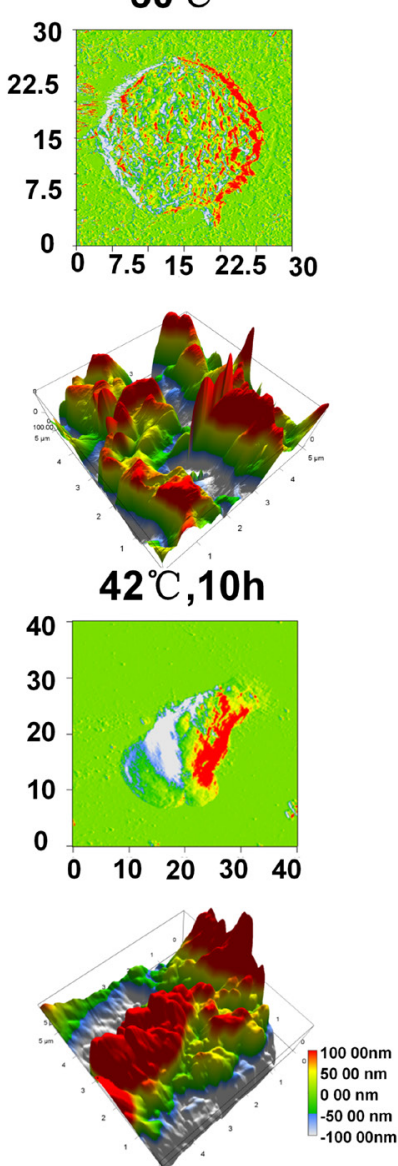

Figure 7. Ultrastructure of cellular membrane imaged by AFM. The deflection image shows the profile of a single cell at various temperatures. The topographic images show the height distribution of the membrane with the size of $5 \mu \mathrm{m} \times 5 \mu \mathrm{m}$. For $37^{\circ} \mathrm{C}$ and $42^{\circ} \mathrm{C}$, the surface of membrane was relatively flat and no pore-like structures were detected after MHIFU treatment immediately. More protruding structures can be observed at $50{ }^{\circ} \mathrm{C}$. After $4 \mathrm{~h}$ MHIFU-mediated drug delivery at 42 ${ }^{\circ} \mathrm{C}$, the membrane surface was full of squamous structures with more uniform size.

\section{Conclusions}

We have demonstrated that a SAW-based microfluidic chip is capable of mimicking the bulk HIFU system and regulating the temperature elevation precisely to control the release of drug from TSL. The disposable nature of the device not only reduces the high cost of the piezoelectric wafer, but also facilitates the subsequent procedures, such as flow cytometry analysis and AFM scanning. Simultaneous quantitative analysis of drug uptake and apoptosis revealed that there is a synergy effect between the ultrasound 
and the drug release at $42{ }^{\circ} \mathrm{C}$ promoting apoptosis. Our study suggests that besides thermal effects, mechanical effects, such as acoustic streaming, play a role in the drug delivery. The MHIFU has the cumulative advantages of precise temperature control, disposable cavity, biocompatibility, small size, low cost and no need for a bulky ultrasonic transmission system. These features may afford insight into the mechanism of the HIFU-mediated drug delivery and provide a versatile toolbox for biomedical applications in drug discovery.

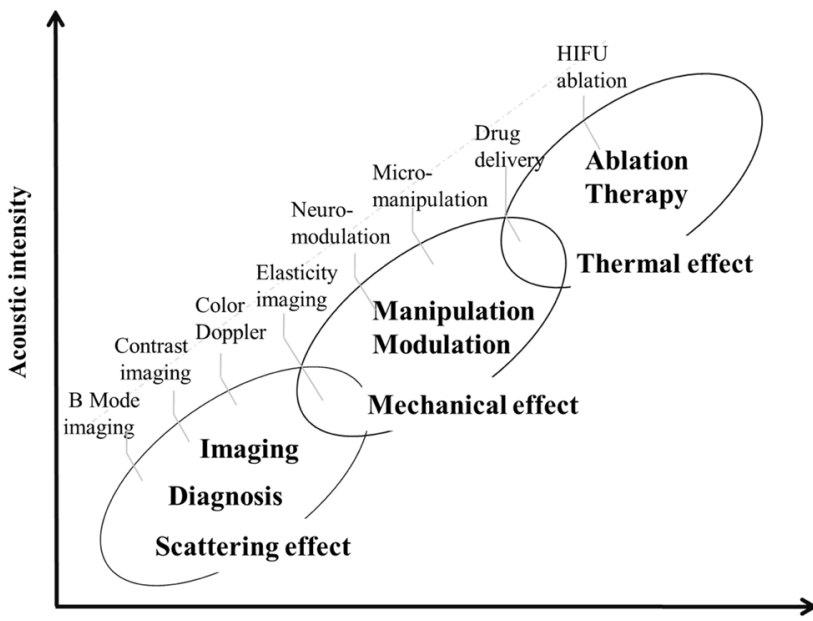

Irradiation time

Figure 8. Three primary acoustic effects of ultrasound and their biomedical applications.

\section{Supplementary Material}

Additional File 1:

Movie S1. http:/ / www.thno.org/v05p1203s1.avi

Additional File 2:

Movie S2. http:/ /www.thno.org/v05p1203s2.avi

Additional File 3:

Movie S3. http:/ / www.thno.org/v05p1203s3.avi

Additional File 4:

Figures S1-S3, Table S1.

http://www.thno.org/v05p1203s4.pdf

\section{Acknowledgements}

The work was supported by 973 Program (Grant No. 2015CB755500) and National Natural Science Foundation of China (Grant Nos. 11304341, 11302239, 11274008, 81401435. and 11325420). Guangdong Research Grant (S2013040014610), Shenzhen Basic Science Research (JCYJ20140417113430580, and JCYJ20130402113127516), and Shenzhen Key Laboratory of Nanobiomechanics.

\section{Competing Interests}

The authors have declared that no competing interest exists.

\section{References}

1. Wagner AD, Grothe W, Haerting J, Kleber G, Grothey A, Fleig WE. Chemotherapy in advanced gastric cancer: a systematic review and meta-analysis based on aggregate data. J Clin Oncol. 2006; 24: 2903-9.

2. Farokhzad OC, Cheng J, Teply BA, Sherifi I, Jon S, Kantoff PW, et al. Targeted nanoparticle-aptamer bioconjugates for cancer chemotherapy in vivo. Proc Nat Acad Sci USA. 2006; 103: 6315-20.

3. Gabizon A, Papahadjopoulos D. Liposome formulations with prolonged circulation time in blood and enhanced uptake by tumors. Proc Nat Acad Sci USA. 1988; 85: 6949-53.

4. Jin L, Zeng X, Liu M, Deng Y, He N. Current progress in gene delivery technology based on chemical methods and nano-carriers. Theranostics. 2014; 4: 240.

5. Chen W, Meng F, Cheng R, Zhong Z. pH-Sensitive degradable polymersomes for triggered release of anticancer drugs: A comparative study with micelles. J Control Release. 2010; 142: 40-6.

6. Paasonen L, Laaksonen T, Johans C, Yliperttula M, Kontturi K, Urtti A. Gold nanoparticles enable selective light-induced contents release from liposomes. J Control Release. 2007; 122: 86-93.

7. Mura S, Nicolas J, Couvreur P. Stimuli-responsive nanocarriers for drug delivery. Nat Mater. 2013; 12: 991-1003.

8. Leung SJ, Romanowski M. Light-activated content release from liposomes. Theranostics. 2012; 2: 1020 .

9. Dreher MR, Liu W, Michelich CR, Dewhirst MW, Chilkoti A. Thermal cycling enhances the accumulation of a temperature-sensitive biopolymer in solid tumors. Cancer Res. 2007; 67: 4418-24.

10. Jing L, Liang X, Li X, Lin L, Yang Y, Yue X, et al. Mn-porphyrin conjugated Au nanoshells encapsulating doxorubicin for potential magnetic resonance imaging and light triggered synergistic therapy of cancer. Theranostics. 2014; 4 : 858 .

11. Dalecki D. Mechanical bioeffects of ultrasound. Annu Rev Biomed Eng. 2004; 6: 229-48.

12. Kennedy JE. High-intensity focused ultrasound in the treatment of solid tumours. Nat Rev Cancer. 2005; 5: 321-7.

13. Ter Haar G. Acoustic surgery. Phys Today. 2001; 54: 29-34.

14. Nyborg WLM, Wu J. Emerging therapeutic ultrasound: World Scientific; 2006.

15. Ranjan A, Jacobs GC, Woods DL, Negussie AH, Partanen A, Yarmolenko PS, et al. Image-guided drug delivery with magnetic resonance guided high intensity focused ultrasound and temperature sensitive liposomes in a rabbit Vx2 tumor model. J Control Release. 2012; 158: 487-94.

16. Mitragotri S. Healing sound: the use of ultrasound in drug delivery and other therapeutic applications. Nat Rev Drug Discov. 2005; 4: 255-60.

17. Liu H-L, Fan C-H, Ting C-Y, Yeh C-K. Combining microbubbles and ultrasound for drug delivery to brain tumors: current progress and overview. Theranostics. 2014; 4: 432 .

18. Meng L, Cai F, Chen J, Niu L, Li Y, Wu J, et al. Precise and programmable manipulation of microbubbles by two-dimensional standing surface acoustic waves. Appl Phys Lett. 2012; 100: 173701.

19. Meng L, Cai F, Zhang Z, Niu L, Jin Q, Yan F, et al. Transportation of single cell and microbubbles by phase-shift introduced to standing leaky surface acoustic waves. Biomicrofluidics. 2011; 5: 044104.

20. Meng L, Cai F, Jin Q, Niu L, Jiang C, Wang Z, et al. Acoustic aligning and trapping of microbubbles in an enclosed PDMS microfluidic device. Sens Actuators B: Chem. 2011; 160: 1599-605.

21. Shi J, Mao X, Ahmed D, Colletti A, Huang TJ. Focusing microparticles in a microfluidic channel with standing surface acoustic waves (SSAW). Lab Chip. 2007; 8: 221-3.

22. Ding X, Peng Z, Lin S-CS, Geri M, Li S, Li P, et al. Cell separation using tilted-angle standing surface acoustic waves. Proc Nat Acad Sci USA. 2014; 111: 12992-7.

23. Zhang J, Meng L, Cai F, Zheng H, Courtney CRP. Multi-scale patterning of microparticles using a combination of surface acoustic waves and ultrasonic bulk waves. Appl Phys Lett. 2014; 104.

24. Reboud J, Bourquin Y, Wilson R, Pall GS, Jiwaji M, Pitt AR, et al. Shaping acoustic fields as a toolset for microfluidic manipulations in diagnostic technologies. Proc Nat Acad Sci USA. 2012.

25. Tan MK, Friend JR, Yeo LY. Interfacial Jetting Phenomena Induced by Focused Surface Vibrations. Phys Rev Lett. 2009; 103: 024501.

26. Li H, Friend JR, Yeo LY. Surface acoustic wave concentration of particle and bioparticle suspensions. Biomed Microdevices. 2007; 9: 647-56.

27. Qi A, Yeo L, Friend J, Ho J. The extraction of liquid, protein molecules and yeast cells from paper through surface acoustic wave atomization. Lab Chip. 2010; 10: 470.

28. Qi A, Friend JR, Yeo LY, Morton DA, McIntosh MP, Spiccia L. Miniature inhalation therapy platform using surface acoustic wave microfluidic atomization. Lab Chip. 2009; 9: 2184-93.

29. Meng L, Cai F, Jiang P, Deng Z, Li F, Niu L, et al. On-chip targeted single cell sonoporation with microbubble destruction excited by surface acoustic waves. Appl Phys Lett. 2014; 104.

30. Needham D, Anyarambhatla G, Kong G, Dewhirst MW. A new temperature-sensitive liposome for use with mild hyperthermia: characterization and testing in a human tumor xenograft model. Cancer Res. 2000; 60: 1197-201. 
31. Niu L, Qian $\mathrm{M}$, Wan $\mathrm{K}, \mathrm{Yu} \mathrm{W}$, Jin $\mathrm{Q}$, Ling $\mathrm{T}$, et al. Ultrasonic particle image velocimetry for improved flow gradient imaging: algorithms, methodology and validation. Phys Med Biol. 2010; 55: 2103.

32. Malek AM, Alper SL, Izumo S. Hemodynamic shear stress and its role in atherosclerosis. J Am Med Assoc. 1999; 282: 2035-42.

33. Sritharan K, Strobl CJ, Schneider MF, Wixforth A, Guttenberg Z. Acoustic mixing at low Reynold's numbers. Appl Phys Lett. 2006; 88: 054102.

34. Lentacker I, De Cock I, Deckers R, De Smedt SC, Moonen CTW. Understanding ultrasound induced sonoporation: Definitions and underlying mechanisms. Adv Drug Delivery Rev. 2013.

35. Tyler WJ. Noninvasive neuromodulation with ultrasound? A continuum mechanics hypothesis. Neuroscientist. 2011; 17: 25-36

36. Tyler WJ, Tufail Y, Pati S. Noninvasive functional neurosurgery using ultrasound. Nat Rev Neurosci. 2008; 9: 768-78.

37. Ding X, Lin S-CS, Kiraly B, Yue H, Li S, Chiang I-K, et al. On-chip manipulation of single microparticles, cells, and organisms using surface acoustic waves. Proc Nat Acad Sci USA. 2012.

38. Franke T, Abate AR, Weitz DA, Wixforth A. Surface acoustic wave (SAW) directed droplet flow in microfluidics for PDMS devices. Lab Chip. 2009; 9: 2625-7.

39. Wu J, Nyborg WL. Ultrasound, cavitation bubbles and their interaction with cells. Adv Drug Delivery Rev. 2008; 60: 1103-16.

40. Lum AF, Borden MA, Dayton PA, Kruse DE, Simon SI, Ferrara KW. Ultrasound radiation force enables targeted deposition of model drug carriers loaded on microbubbles. J Control Release. 2006; 111: 128-34.

41. Jin H, Zhou J, He X, Wang W, Guo H, Dong S, et al. Flexible surface acoustic wave resonators built on disposable plastic film for electronics and lab-on-a-chip applications. Sci Rep. 2013; 3. 